\section{Positiver Hämoccult: Schnelle Koloskopie!}

Zwischen einem positiven Hämoccult und der koloskopischen Abklärung sollte nicht allzu viel Zeit verstreichen, zeigt eine US-Studie. Verglichen mit einer endoskopischen Untersuchung an den Tagen 8-30 brachte ein Aufschub bis zum sechsten Monat keine Unterschiede in der Häufigkeit von Karzinomen. Bis zum zehnten Monat war nur ein Zuwachs bei Karzinomen im Stadium II zu verzeichnen. Erfolgte die Darmspiegelung jedoch erst nach zehn Monaten, war das Risiko für Karzinome in allen Stadien deutlich erhöht. Wurde länger als zwölf Monate gewartet, waren Karzinome insgesamt sogar mehr als doppelt und fortgeschrittene Karzinome gut dreimal so häufig wie bei der frühen Koloskopie.

JAMA 2017;317(16):1631-1641

\section{Softdrinks als Gefahr für das Gehirn}

Der Konsum von Softdrinks mit künstlichen Süßstoffen geht mit einem höheren Risiko für Schlaganfälle und Demenz einher, wie eine Studie aus den USA ergeben hat. Knapp 3.000 Probanden waren regelmäßig zu ihren Trinkgewohnheiten befragt worden. 97 Teilnehmer erlitten einen Schlaganfall, 81 erkrankten an einer Demenz. Der Konsum von künstlich gesüßten Softdrinks war signifikant mit beiden Erkrankungen assoziiert. Wer bis zu sieben Jahre lang mindestens einmal täglich zu solchen Getränken griff, hatte ein 2,6-fach erhöhtes Schlaganfallrisiko und ein 2,89-fach höheres Risiko, an Alzheimer zu erkranken.

Stroke 2017; online 24. April

\section{Asthma erhöht das Infektionsrisiko}

Patienten, die vor dem 50. Lebensjahr an Asthma erkranken, haben ein erhöhtes Risiko für gravierende Infektionen, stellten dänische Autoren fest. An der Untersuchung waren über 105.000 Erwachsene beteiligt. Während der Beobachtungszeit von median sechs Jahren betraf dies 11.160 Personen. Die 6.750 Teilnehmer mit Asthma hatten im Vergleich zu denjenigen ohne atopische Erkrankung ein um 54\% erhöhtes Infektionsrisiko.

J Intern Med 2017, 26. Juli; doi: 10.1111/ joim. 12635

\title{
Ist Stress ein Grund für Pickel nach dem Teenageralter?
}

\begin{abstract}
Wenn Frauen im Alter über 25 (noch) an Akne leiden, hängt das möglicherweise auch mit ihrer Lebensführung zusammen. Die Risikofaktoren scheinen sich aber von denen einer Pubertätsakne zu unterscheiden.
\end{abstract}

$V_{s}$ on 20,5 auf 26,5 Jahre ist das Durchschnittsalter von Aknepatienten in den vergangenen Jahrzehnten gestiegen. Warum vor allem Frauen immer häufiger im Alter über 25 Jahren noch oder erstmals von Akne betroffen sind, ist ungeklärt. In einer Fall-Kontroll-Studie wurden nun mögliche Verursacher ausfindig gemacht. 248 konsekutive Patientinnen aus acht dermatologischen Klinikambulanzen in Italien mit persistierender oder Late-Onset-Akne nahmen an der Studie teil. Als Kontrollen dienten 270 Patientinnen mit anderen Hauterkrankungen.

Bei den meisten Aknepatientinnen (mittleres Alter, 32 Jahre) war die Erkrankung leicht (50\%) oder mittelschwer (42\%) ausgeprägt. Bei 75\% handelte es sich um die entzündliche Form, bei knapp einem Drittel war neben Gesicht auch der Körperstamm betroffen. Erwartungsgemäß hatten die Aknepatientinnen bereits in der Adoleszenz häufiger an Akne gelitten und öfter eine positive Familienanamnese. Altersadjustiert waren die beiden Faktoren mit einem rund fünf- bzw. dreimal so hohen Risiko einer späten Akne assoziiert.

\section{Psychische Belastungen als mögliche Ursache}

Frauen mit Akne waren seltener schon schwanger gewesen. Außerdem wurde bei ihnen häufiger Hirsutismus diagnostiziert. Beides sind Hinweise auf einen möglichen hormonellen Hintergrund der Acne tarda, etwa eine Hyperandrogenämie. Beim Gebrauch oraler Kontrazeptiva gab es aber keinen Unterschied zur Kontrollgruppe. Auch in früheren Studien war nur ein schwacher Zusammenhang mit Androgenanomalien gefunden worden. Häufiger als die Kontrollgruppe waren die Aknepatientinnen als Büroangestellte tätig. Ihren psychischen Stress im letzten Monat empfanden sie öfter als hoch oder sehr hoch.
Die Assoziation mit Stress halten die Studienautoren für besonders interessant. Auch in anderen Studien seien Verbindungen von Akne im Erwachsenenalter mit psychischen Erkrankungen wie Depressionen und Somatisierungsstörungen beschrieben worden. Psychische Faktoren seien möglicherweise auch ein Grund, warum Büroangestellte öfter als etwa Hausfrauen von Akne betroffen waren.

In Bezug auf die Ernährungsgewohnheiten unterschieden sich Aknepatientinnen von der Vergleichsgruppe durch einen geringeren Verzehr von Obst und Gemüse sowie von Fisch. Im Gegensatz $\mathrm{zu}$ früheren Studien zur Pubertätsakne bestand aber kein Zusammenhang mit dem Konsum von Milch(produkten). Auch der Verzehr von Kuchen und Süßigkeiten, Schokolade oder Rindfleisch hatte keine erkennbaren Auswirkungen auf das Aknerisiko. Di Landro et al. interpretieren das als Hinweis auf eine von der Adoleszentenakne abweichende $\mathrm{Pa}$ thogenese, bei der metabolische Faktoren einen geringeren Einfluss haben. Dazu passt auch, dass die Acne tarda nicht mit einem erhöhten BMI vergesellschaftet war. Auch ein immer wieder diskutierter Zusammenhang mit dem Rauchen konnte nicht bestätigt werden.

Die Studie sagt natürlich nichts über die Kausalität der festgestellten Zusammenhänge. „Zumindest teilweise können die Assoziationen auch gemeinsame Risikofaktoren oder Konsequenzen einer bestehenden Akne widerspiegeln", so die Autoren. Dennoch empfehlen sie, beim Management von Frauen mit Acne tarda „Lebensstilfaktoren angemessen zu berücksichtigen“.

(bs)

Di Landro A et al. Adult female acne and associated risk factors: Results of a multicenter casecontrol study in Italy. J Am Acad Dermatol. 2016 Dec;75(6):1134-41.e1 